\title{
Effects of sterol derivatives in cationic liposomes on biodistribution and gene-knockdown in the lungs of mice systemically injected with siRNA lipoplexes
}

\author{
YOSHIYUKI HATTORI ${ }^{1}$, HIROMU SAITO ${ }^{1}$, TERUAKI OKU ${ }^{2}$ and KEI-ICHI OZAKI ${ }^{3}$ \\ Departments of ${ }^{1}$ Molecular Pharmaceutics and ${ }^{2}$ Microbiology, Hoshi University, Tokyo 142-8501; \\ ${ }^{3}$ Department of Molecular Pathology, Faculty of Pharmaceutical Sciences, \\ Doshisha Women's College of Liberal Arts, Kyotanabe, Kyoto 610-0395, Japan
}

Received March 25, 2021; Accepted June 4, 2021

DOI: $10.3892 / \mathrm{mmr} .2021 .12237$

\begin{abstract}
Cationic liposomes can be intravenously injected to deliver short interfering (si)RNAs into the lungs. The present study investigated the effects of sterol derivatives in systemically injected siRNA/cationic liposome complexes (siRNA lipoplexes) on gene-knockdown in the lungs of mice. Cationic liposomes composed of 1,2-dioleoyl-3-trimethylammonium-propane or dimethyldioctadecylammonium bromide (DDAB) were prepared as a cationic lipid, with sterol derivatives such as cholesterol (Chol), $\beta$-sitosterol, ergosterol (Ergo) or stigmasterol as a neutral helper lipid. Transfected liposomal formulations composed of DDAB/Chol or DDAB/Ergo did not suppress the expression of the luciferase gene in LLC-Luc and Colon 26-Luc cells in vitro, whereas other formulations induced moderate gene-silencing. The systemic injection of siRNA lipoplexes formulated with Chol or Ergo into mice resulted in abundant siRNA accumulation in the lungs. In comparison, systemically injected DDAB/Chol or DDAB/Ergo lipoplexes of Tie 2 siRNA effectively increased the suppression of the Tie2 mRNA expression in the lungs of mice. These findings indicated that DDAB/Chol and DDAB/Ergo liposomes could function as vectors for siRNA delivery to the lungs.
\end{abstract}

\section{Introduction}

Small interfering RNA (siRNA) therapeutics are a new class of drugs comprising synthetic RNA duplexes that target a specific mRNA for degradation. The lungs are promising targets of siRNA therapy in terms of pathological states such as infectious diseases, asthma, and cancer $(1,2)$. Therapy with siRNA reduced the formation of lung metastases and increased

Correspondence to: Professor Yoshiyuki Hattori, Department of Molecular Pharmaceutics, Hoshi University, 2-4-41 Ebara, Shinagawa, Tokyo 142-8501, Japan

E-mail: yhattori@hoshi.ac.jp

Key words: cationic liposome, sterol derivative, short interfering RNA delivery, lung, gene-knockdown the life span of mouse models of lung metastasis (3), although few siRNA therapeutics are in advanced clinical trials for pulmonary diseases. The main routes of administering siRNA therapeutics for pulmonary delivery are inhalation and intravenous injection (4). Inhalation is less invasive and the lung epithelium can be targeted. However, several barriers must be surmounted, such as being swept away by respiratory cilia and the need to penetrate the pulmonary mucus layer (5). The intravenous administration of siRNA therapeutics is limited by instability in the blood circulation, ineffective delivery to the target, and the potential for renal and hepatic toxicity. These limitations can be addressed using siRNA delivery systems for targeting to the lungs via systemic injection. Cationic liposomes have recently attracted attention as a means of delivering siRNA to the lungs. The systemic injection with cationic liposomes aids successful siRNA delivery to the lungs $(1,5)$ and induces the specific gene silencing in pulmonary endothelial cells $(6,7)$. Cationic liposomes consisting of the cationic lipid 1,2-dioleoyl-3-trimethylammonium-propane (DOTAP) have served as vectors for siRNA delivery to the pulmonary epithelium and endothelium via systemic injection (6). Previously, for evaluation of gene-silencing efficacy in lung, we used 11 kinds of dialkyl or trialkyl cationic lipids and 6 kinds of cationic cholesterol derivatives as cationic lipids, and prepared 17 kinds of cationic liposomes composed of a type of cationic lipid and neutral helper lipid (1,2-dioleoyl-L- $\alpha$-gl ycero-3-phosphatidylethanolamine, DOPE) (8). We found that cationic liposomes composed of dimethyldioctadecylammonium bromide (DDAB) and DOPE resulted in abundant siRNA accumulation in the lung and the significant suppression of targeted mRNA expression after systemic injection of siRNA lipoplexes (8). We also showed that including cholesterol (Chol) in DDAB or DOTAP liposomes increased the accumulation of systemically injected siRNA lipoplexes and gene silencing effects in the lungs compared with DOPE (9). However, to the best of our knowledge, the effects of sterol derivatives in liposomal formulations containing cationic liposomes for siRNA delivery to the lungs have never been determined.

Here, we examined the effects of sterol derivatives in cationic liposomes on siRNA delivery to the lungs. We systemically injected siRNA lipoplexes comprising the cationic lipids 
DOTAP and DDAB combined with Chol, $\beta$-sitosterol (Sito), ergosterol (Ergo), or stigmasterol (Stig), then evaluated the biodistribution and gene knockdown effects in the lungs. The systemically injected DDAB/Chol or DDAB/Ergo lipoplexes resulted in efficient gene-silencing in the lungs.

\section{Materials and methods}

Materials. We obtained the following from the respective suppliers: 1,2-dioleoyl-3-trimethylammonium-propane methyl sulfate salt (DOTAP; Avanti Polar Lipids Inc.), dimethyldioctadecylammonium bromide (DDAB, product name: DC-1-18; Sogo Pharmaceutical Co., Ltd.), cholesterol (Chol; Wako Pure Chemical Industries, Ltd.), $\beta$-sitosterol (Sito; Tama Biochemical Co., Ltd.), Ergosterol (Ergo) and stigmasterol (Stig; Tokyo Chemical Industry Co., Ltd.). All other chemicals were of the highest available grade.

Small interfering RNAs. The siRNAs targeting the nucleotides of pGL3 and pGL4 Firefly luciferase siRNA (Luc siRNA), negative control (Cont) siRNA for Luc siRNA, and cyanine 5.5 (Cy5.5)-labeled siRNA (Cy5.5-siRNA) were synthesized by Sigma Genosys. Mouse Tie2 siRNA and luciferase siRNA (Cont siRNA) as a negative control for Tie2 siRNA were obtained from Japan Bio Services Co., Ltd. The siRNA sequences of the pGL3 Luc siRNA, pGL4 Luc siRNA, and Cont siRNA for Luc siRNA are described elsewhere $(10,11)$, as is Cy5.5-siRNA (12). Tie2 siRNA and Cont siRNA as a negative control for Tie2 siRNA were alternating 2'-O-methyl-modified, blunt-ended siRNA, and siRNA sequences as described $(3,13)$.

Preparation of cationic liposomes and siRNA lipoplexes. Cationic liposomes were prepared from cationic lipid/sterol derivatives at a molar ratio of 1:1. Cationic lipid and sterol derivatives were dissolved in chloroform to prepare cationic liposomes using thin-film hydration. The chloroform was then evaporated under vacuum on a rotary evaporator at $60^{\circ} \mathrm{C}$ to obtain a thin film, which was then hydrated with water at $60^{\circ} \mathrm{C}$ by vortex mixing. The liposomes were disrupted in a Bransonic $^{\circledR} 2510 \mathrm{~J}-\mathrm{MTH}, 100 \mathrm{~W}$ bath-type sonicator (Branson Utrasonics Corp.) for 5-10 min at room temperature.

Liposomes were vortex-mixed for $10 \mathrm{sec}$ with siRNA at a charge ratio (+:-) of 4:1, then incubated at room temperature for $15 \mathrm{~min}$ to prepare siRNA/cationic liposome complexes (siRNA lipoplexes). The charge ratio (+:-) of cationic liposomes to siRNA is expressed as the molar ratio of cationic lipid to siRNA phosphate.

Size and $\zeta$-potential of cationic liposomes and siRNA lipoplexes. The particle size distributions and $\zeta$-potentials of cationic liposomes and siRNA lipoplexes were measured using an ELS-Z2 light-scattering photometer (Otsuka Electronics Co., Ltd.) at $25^{\circ} \mathrm{C}$ after dispersion in $\sim 1.5 \mathrm{ml}$ of water as described (14).

Free siRNA in siRNA lipoplex suspensions. Lipoplexes carrying siRNA ( $1 \mu \mathrm{g})$ were formed at charge ratios (+:-) of 1:1,2:1,3:1 and 4:1. Amounts of free siRNA in siRNA lipoplex suspensions were measured using exclusion assays with SYBR ${ }^{\circledR}$ Green I Nucleic Acid Gel Stain (Takara Bio Inc.) as described (15). The fluorescence value obtained by adding free siRNA was set as $100 \%$.
Cell culture. Murine colon adenocarcinoma Colon 26 cells stably expressing pGL3-luciferase (Colon 26-Luc) were donated by Professor Takashi Murakami (Department of Microbiology, Saitama Medical University, Saitama, Japan) (16). Mouse Lewis lung carcinoma LLC cells were obtained from the Cell Resource Center for Biomedical Research, Institute of Development, Aging, and Cancer, Tohoku University (Sendai, Japan). The cells were cultured in RPMI-1640 medium with $10 \%$ heat-inactivated fetal bovine serum (FBS; Invitrogen; Thermo Fisher Scientific, Inc.) and kanamycin $(100 \mu \mathrm{g} / \mathrm{ml})$ at $37^{\circ} \mathrm{C}$ in a $5 \% \mathrm{CO}_{2}$ humidified atmosphere.

A DNA construct for Luc2 expression was amplified by PCR using plasmidDNA encoding the pGL4 luciferase gene (Promega Corp.). This construct served as a template to prepare LLC cells stably expressing pGL4-luciferase (LLC-Luc). The following primers were included in the PCR reaction: 5'-ATTTCCGGTGA ATTCATGGAAGATGCCAAAAAC-3' and 5'-AGTCTCG AGGAATTCTTACACGGCGATCTTGCC-3'. The product was cloned into the EcoRI restriction site of the pLVSIN-CMV Pur vector (pLVSIN-Luc2; Takara Bio Inc.) using the In-Fusion HD cloning system (Takara Bio Inc.). Lentiviral particles were co-transfected with pLVSIN-Luc2 and ViraPower (three packaging plasmids, Invitrogen) using polyethylenimine 'MAX' (Polysciences Inc.), produced in 293FT cells (Invitrogen; Thermo Fisher Scientific, Inc.), and concentrated by PEG precipitation as described $(17,18)$. The virus solution was mixed with hexadimethrine bromide (final concentration, $8 \mu \mathrm{g} / \mathrm{ml}$ ), then the mixture was added to cultured LLC cells $\left(1 \times 10^{5} /\right.$ well in 6-well plates). The plates were centrifuged $(1,800 \mathrm{x} \mathrm{g}, 90 \mathrm{~min})$ at $32^{\circ} \mathrm{C}$ and incubated at $37^{\circ} \mathrm{C}$ for $24 \mathrm{~h}$. The medium was replaced with RPMI-1640 medium supplemented with 10\% FBS cells, then the cells were successively cultured for $48 \mathrm{~h}$ by selection with puromycin $(2.5 \mu \mathrm{g} / \mathrm{ml})$.

Gene-silencing effects of siRNA lipoplexes in cultured cells. We seeded LLC-Luc or Colon 26-Luc cells $\left(3 \times 10^{5} /\right.$ well) in 6-well plates before transfection. siRNA lipoplexes were formed at a charge ratio (+:-) of 4:1 by vortex-mixing cationic liposomes with 50 pmol of Cont siRNA or Luc siRNA for $10 \mathrm{sec}$, then incubating the mixture at room temperature for $15 \mathrm{~min}$. The cells were then transfected with siRNA lipoplexes diluted in $1 \mathrm{ml}$ of medium supplemented with $10 \%$ FBS (final $50 \mathrm{nM}$ siRNA concentration). Forty-eight hours after transfection, the cells were lysed by addition of $250 \mu \mathrm{l}$ of cell lysis buffer (Pierce ${ }^{\mathrm{TM}}$ Luciferase Cell Lysis Buffer; Thermo Fisher Scientific, Inc.) after washing with PBS, and subjected to one cycle of freezing $\left(-80^{\circ} \mathrm{C}\right)$ and thawing at $37^{\circ} \mathrm{C}$, followed by centrifugation at $15,000 \mathrm{x}$ g for $10 \mathrm{sec}$. Aliquots $(10 \mu \mathrm{l})$ of supernatants were mixed with $50 \mu \mathrm{l}$ of PicaGene MelioraStar-LT Luminescence Reagent (Toyo Ink Mfg. Co. Ltd.), and luminescence was measured as described (14). The protein concentration in the supernatants was determined using Pierce ${ }^{\mathrm{TM}}$ BCA Protein Assay Kits (Thermo Fisher Scientific, Inc.). Luciferase activity (\%) was calculated relative to the luciferase activity (cps/ $\mu \mathrm{g}$ protein) of untransfected cells.

Cytotoxicity of siRNA lipoplexes. Colon 26-Luc or LLC-Luc cells were seeded in 96-well plates $24 \mathrm{~h}$ before transfection. Each siRNA lipoplex with 50 pmol Cont siRNA was diluted in $1 \mathrm{ml}$ of medium supplemented with $10 \%$ FBS. The mixture (100 $\mu \mathrm{l})$ was then added to the cells at $50 \%$ confluency in the 
well (final $50 \mathrm{nM}$ siRNA concentration). After a $24 \mathrm{~h}$ incubation period, cell numbers were determined using a Cell Counting Kit-8 (Dojindo Laboratories). Cell viability was expressed as relative to the absorbance at $450 \mathrm{~nm}$ of untransfected cells.

Agglutination assays. Erythrocytes were separated as described (14) from whole blood $(0.3 \mathrm{ml})$ collected from the jugular vein of an 8-week-old female BALB/c mouse (Sankyo Labo Service Corp., Inc.) under anesthesia induced by isoflurane inhalation (4\% for induction and $1.5-2 \%$ for maintenance; FUJIFILM Wako Pure Chemical Corporation). Lipoplexes containing siRNA $(2 \mu \mathrm{g})$ were then incubated with $100 \mu \mathrm{l}$ of $2 \%(\mathrm{v} / \mathrm{v})$ erythrocyte suspension for $15 \mathrm{~min}$ at $37^{\circ} \mathrm{C}$, then samples on glass plates were assessed by phase-contrast microscopy.

Biodistribution of siRNA after systemic injection of siRNA lipoplexes into mice. All animal experiments were conducted in accordance with 'Guide for the Care and Use of Laboratory Animals' published by the U.S. National Institutes of Health and 'Guide for the Care and Use of Laboratory Animals' adopted by the Institutional Animal Care and Use Committee of Hoshi University (Tokyo, Japan), which is accredited by the Ministry of Education, Culture, Sports, Science, and Technology, Japan. The Institutional Animal Care and Use Committee of Hoshi University approved this study (permission no. 20-018).

Seven female BALB/c mice (18-20 g, 8 weeks of age; Sankyo Labo Service Corp. Inc.) were housed at $24^{\circ} \mathrm{C}$ and $55 \%$ humidity with a $12 / 12 \mathrm{~h}$ light/dark cycle (lights on at 8:00 a.m.) and access to food and water ad libitum. Lipoplexes carrying $20 \mu \mathrm{g}$ of Cy5.5-siRNA were injected into the lateral tail veins of mice ( $n=1$ per siRNA lipoplex), then all mice were sacrificed by cervical dislocation $1 \mathrm{~h}$ later as described (19), and cervical dislocation was confirmed by careful assessment of the mice for explicit signs of death such as cardiac arrest. Tissues were analyzed by Cy5.5 fluorescence imaging using a NightOWL LB981 NC100 system (Berthold Technologies GmbH \& Co. KG., Bad Wildbad, Germany) as described (14).

Tie 2 mRNA levels in the lungs after systemically injecting mice with siRNA lipoplexes. Lipoplexes with 5, 10 or $20 \mu \mathrm{g}$ of Cont siRNA or Tie2 siRNA were injected into the lateral tail veins of 8-week-old female BALB/c mice ( $n=3-4$ per siRNA lipoplex), then Tie 2 mRNA expression in the lungs was analyzed $48 \mathrm{~h}$ later. Complementary DNA was synthesized from total RNA isolated using Isogen II (Nippon Gene Co., Ltd.), then real-time quantitative PCR proceeded using TaqMan Gene expression assays (Tek [Tie-2], Mm00443243_m1, phosphatase, and tensin homolog [PTEN]: Mm00477208_m1; Applied Biosystems ${ }^{\circledR}$ ) on a Roche Light Cycler 96 system. Levels of Tie2 mRNA expression were normalized to those of PTEN mRNA in the same sample as described (3) and analyzed using the comparative $\mathrm{Cq}\left(2^{-\Delta \Delta \mathrm{Cq}}\right)$ method (20).

Determination of serum transaminase activity. Lipoplexes carrying $20 \mu \mathrm{g}$ of Cont siRNA were injected into the lateral tail veins of mice ( $n=4$ per siRNA lipoplex), then serum was separated from whole blood collected from the mice and coagulated $24 \mathrm{~h}$ later. We then measured aspartate aminotransferase (AST/GOT) and alanine aminotransferase (ALT/GPT) levels in the serum using Transaminase CII-test kits (Wako Pure Chemicals). Normal values were determined in blood from age-matched untreated mice.

Statistical analysis. Data are presented as means \pm standard deviation (SD) of triplicate determinations. The statistical significance of differences between means was determined by Student t-tests using GraphPad Prism 4.0 (GraphPad Software Inc.). Multiple comparisons were assessed by analysis of variance followed by one-way analysis of variance on ranks with post-hoc Tukey tests using GraphPad Prism 4.0. Statistical significance was set at $\mathrm{P} \leq 0.05$.

\section{Results and Discussion}

Characterization of cationic liposomes and siRNA lipoplexes. Cationic liposomes comprising dialkyl cationic lipids with neutral helper lipids, such as DOPE or Chol, have been studied extensively as vectors for siRNA delivery $(8,9)$. Here, we investigated the biodistribution and gene knockdown effects of siRNA in the lungs of mice when systemically injected as lipoplexes containing the cationic lipids DOTAP and DDAB, and the sterol derivatives Chol, Stig, Ergo and Sito (Fig. 1). Cholesterol is a major component of animal cell membranes, whereas Ergo is the primary sterol in yeasts and other fungi (21). Both Sito and Stig are derived from plants (22). The main structural difference between Chol and the other three sterols is the presence of extra methyl or ethyl group and/or double bond in side chain of Chol (Fig. 1).

Cationic liposomes were prepared from cationic lipid/sterol derivatives at a molar ratio of 1:1. However, DOTAP/Stig at a molar ratio of 1:1 aggregated during the preparation of cationic liposomes (Table I), so this formulation was excluded from further study. The sizes of cationic liposomes prepared in this study were approximately $100-190 \mathrm{~nm}$. The $\zeta$-potentials were approximately +40-50 $\mathrm{mV}$ (Table I). When the liposomes were mixed with siRNA, their lipoplex sizes were about 160-220 nm and their $\zeta$-potentials were about $+29-38 \mathrm{mV}$ (Table I).

We also examined associations of siRNA with each type of cationic liposome using exclusion assays with $\mathrm{SYBR}^{\circledR}$-Green I. The amount of free siRNA in the siRNA lipoplex suspension was largely decreased by adding DDAB- and DOTAP-cationic liposomes to siRNA beyond a charge ratio (+:-) of 2:1 (Fig. 2). This phenomenon suggested that the cationic liposomes were completely bound to siRNA at this ratio regardless of the sterol derivative.

Effects of sterol derivatives in cationic liposomes on gene knockdown in vitro. We previously showed that including DOPE in cationic liposomal formulations increased, whereas increased Chol content decreased gene-silencing in vitro (9). Here, we investigated the effects of sterol derivatives in cationic liposomes on gene knockdown by siRNA lipoplexes in Colon 26-Luc and LLC-Luc cells. Luciferase activity evaluated using Lipofectamine ${ }^{\mathrm{TM}}$ RNAiMax, revealed that Luc siRNA knocked down $90 \%$ of luciferase genes in LLC-Luc and Colon 26-Luc cells compared with Cont siRNA (data not shown). In contrast, DDAB-based liposomes induced moderate gene silencing when combined with Stig and Sito, but not with Chol and Ergo (Fig. 3A and B). Cholesterol formed a more rigid and ordered liposomal membrane, 

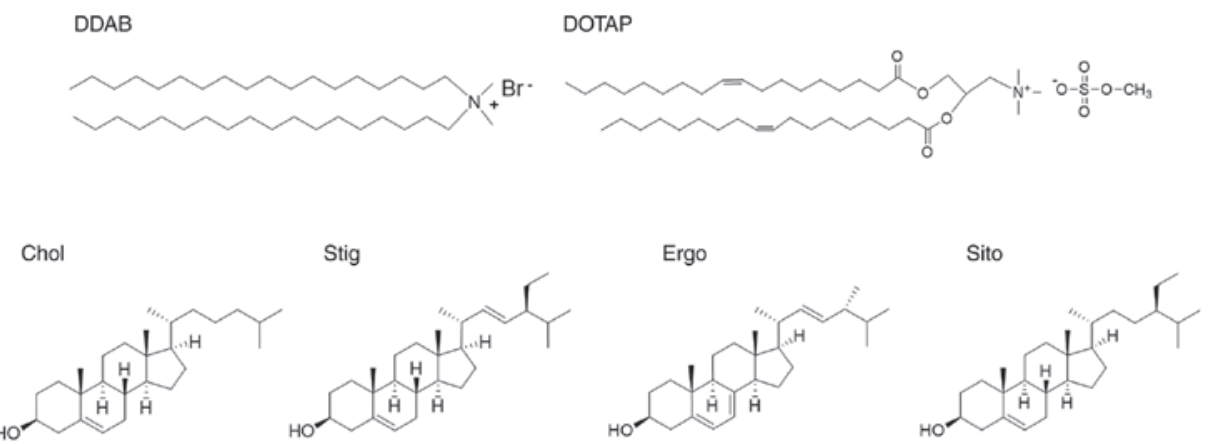

Figure 1. Structure of cationic lipids and sterol derivatives. DDAB, dimethyldioctadecylammonium bromide; DOTAP, 1,2-dioleoyl-3-trimethylammonium-propane methyl sulfate salt; Chol, cholesterol; Stig, stigmasterol; Ergo, ergosterol; Sito, $\beta$-sitosterol.

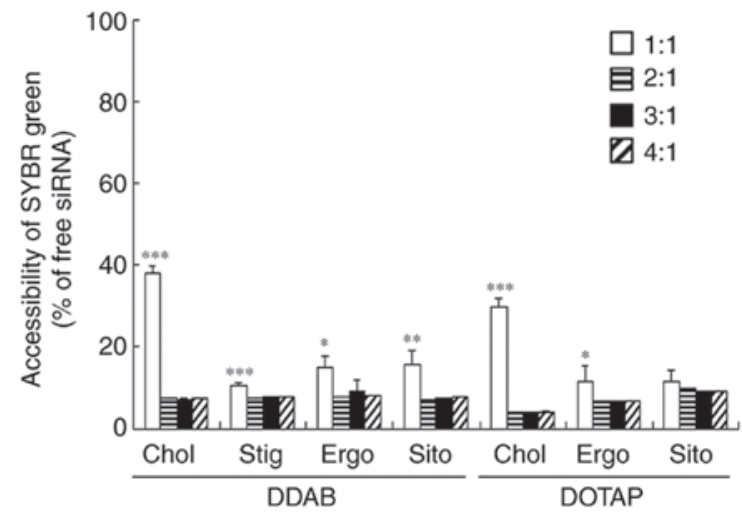

Figure 2. Effects of sterol derivatives in cationic liposomes on the association between siRNA and cationic liposomes. siRNA lipoplexes were formed at charge ratios (+:-) of 1:1, 2:1, 3:1 and 4:1. Amounts of free siRNA in the siRNA lipoplex suspensions were measured using exclusion assays with $\mathrm{SYBR}^{\circledR}$ Green I Nucleic Acid Gel Stain and calculated based on standard curves of free siRNA. Data are presented as the mean \pm standard deviation $(n=3) .{ }^{*} \mathrm{P}<0.05$, ${ }^{* *} \mathrm{P}<0.01$ and ${ }^{* * *} \mathrm{P}<0.001$ vs. charge ratio (+:-) of 4:1. si, short interfering; lipoplex, cationic liposome complexes; DDAB, dimethyldioctadecylammonium bromide; DOTAP, 1,2-dioleoyl-3-trimethylammonium-propane methyl sulfate salt; Chol, cholesterol; Stig, stigmasterol; Ergo, ergosterol; Sito, $\beta$-sitosterol.

suggesting that stabilizing the liposomal membrane might decrease gene-silencing effects by inhibiting the escape of siRNA lipoplexes from endosomes after cellular uptake. In contrast, an extra ethyl group in the side chains of Sito and Stig might enhance the effect of steric hindrance and weaken molecular interactions in DDAB-based liposomal membranes, resulting in improved gene silencing by increasing membrane fluidity. However, DOTAP-based liposomes moderately suppressed luciferase expression regardless of the sterol derivative (Fig. 3A and B). These findings suggested that unsaturation of the dialkyl chains in DOTAP enhances transfection efficiency when combined with sterol derivatives. These results indicated that gene-silencing activities in vitro were affected by the types of cationic lipids and sterol derivatives in the liposomes, although inclusion of sterol derivatives into liposomal formulation did not exhibit strong gene silencing effects in the cells overall.

Cytotoxicity of siRNA lipoplexes. We measured cell viability $24 \mathrm{~h}$ after transfecting Colon 26-Luc and LLC-Luc cells with siRNA lipoplexes to determine the effects of sterol derivatives in cationic liposomes on the cytotoxicity of siRNA lipoplexes.
The lipoplexes were not significantly cytotoxic (>80\% cell viability; Fig. 4).

Interaction with erythrocytes and siRNA lipoplexes. siRNA lipoplexes bound to blood components, such as erythrocytes, in the systemic circulation after systemic administration. Furthermore, agglutinates were captured by lung capillaries $(23,24)$, which are the first microvascular system through which injected siRNA lipoplexes must pass through. We mixed siRNA lipoplexes with erythrocyte suspensions to determine the effects of sterol derivatives in cationic liposomes on their agglutination. All siRNA lipoplexes agglutinated with erythrocyte suspensions, regardless of the sterol derivative in the liposomal formulations (Fig. 5). We did not detect large differences in the appearance of erythrocyte-siRNA lipoplex agglutination regardless of the sterol derivatives in DDAB and DOTAP liposomes.

Biodistribution of siRNA after systemic injection of siRNA lipoplexes. We previously showed that adding Chol to the liposomal formulationincreased siRNA accumulation in the lungs compared with DOPE (9). To investigate the effect of sterol derivatives on the biodistribution of siRNAs after systemic injection of siRNA lipoplexes, mice were systemically injected with lipoplexes carrying Cy5.5-siRNA and the biodistribution of siRNAs was assessed $1 \mathrm{~h}$ later. Injected LP-DDAB/Chol, LP-DDAB/Ergo, LP-DOTAP/Chol, and LP-DOTAP/Ergo lipoplexes induced abundant siRNA accumulation in the lungs (Fig. 6). The inclusion of Chol or Ergo into cationic liposomes resulted in stable aggregates of siRNA lipoplexes with erythrocytes in the blood circulation and efficient capture by lung capillaries. These results indicated that the biodistribution of siRNA after systemic injection of siRNA lipoplexes was largely affected by the sterol derivative in cationic liposomes.

Gene silencing in the lungs after systemic injection of siRNA lipoplexes. The Tie 2 receptor is primarily expressed on endothelial cells $(25,26)$, and the gene-silencing effect of siRNA lipoplexes in the pulmonary endothelium has been evaluated using the Tie2 gene (3). We previously showed that injected LP-DDAB/DOPE lipoplexes carrying $50 \mu \mathrm{g}$ of Tie 2 siRNA significantly suppressed Tie2 mRNA levels in the lungs (79\% knockdown vs. Cont siRNA), whereas LP-DOTAP/DOPE did not (9). We investigated the effects of sterol derivatives in cationic liposomes on knockdown efficiency in the pulmonary endothelium after systemic injection of siRNA lipoplexes. We 
Table I. Particle size and $\zeta$-potential of cationic liposomes and siRNA lipoplexes.

\begin{tabular}{|c|c|c|c|c|c|c|c|}
\hline \multirow[b]{2}{*}{ Liposome } & \multirow[b]{2}{*}{$\begin{array}{c}\text { Formulation, } \\
\text { mol ratio }\end{array}$} & \multicolumn{3}{|c|}{ Liposome } & \multicolumn{3}{|c|}{ Lipoplex $^{b}$} \\
\hline & & $\mathrm{Size}^{\mathrm{a}}, \mathrm{nm}$ & PDI & $\begin{array}{c}\zeta \text {-potential } \\
\mathrm{mV}\end{array}$ & Size $^{\mathrm{a}}, \mathrm{nm}$ & PDI & $\begin{array}{c}\zeta \text {-potential } \\
\mathrm{mV}\end{array}$ \\
\hline LP-DDAB/Chol & DDAB:Chol = 1:1 & $105.1 \pm 0.8$ & $0.27 \pm 0.01$ & $41.5 \pm 0.6$ & $157.6 \pm 1.8$ & $0.22 \pm 0.01$ & $28.7 \pm 1.1$ \\
\hline LP-DDAB/Stig & DDAB:Stig = 1:1 & $110.6 \pm 2.5$ & $0.23 \pm 0.01$ & $50.2 \pm 0.9$ & $214.5 \pm 4.6$ & $0.26 \pm 0.00$ & $31.7 \pm 0.7$ \\
\hline LP-DDAB/Ergo & DDAB:Ergo = 1:1 & $191.0 \pm 19.4$ & $0.17 \pm 0.05$ & $45.7 \pm 1.0$ & $195.2 \pm 2.4$ & $0.20 \pm 0.00$ & $30.6 \pm 1.1$ \\
\hline LP-DDAB/Sito & DDAB: Sito = 1:1 & $100.5 \pm 0.8$ & $0.22 \pm 0.01$ & $47.0 \pm 0.9$ & $223.7 \pm 0.6$ & $0.24 \pm 0.00$ & $27.1 \pm 1.8$ \\
\hline LP-DOTAP/Chol & DOTAP:Chol = 1:1 & $108.7 \pm 0.8$ & $0.24 \pm 0.00$ & $49.1 \pm 1.3$ & $197.1 \pm 1.5$ & $0.23 \pm 0.01$ & $38.3 \pm 0.2$ \\
\hline LP-DOTAP/Stig & DOTAP:Stig = 1:1 & Aggregation & N.D. & N.D. & N.D. & N.D. & N.D. \\
\hline LP-DOTAP/Ergo & DOTAP:Ergo = 1:1 & $148.3 \pm 1.1$ & $0.27 \pm 0.00$ & $40.7 \pm 1.7$ & $171.1 \pm 1.1$ & $0.17 \pm 0.00$ & $38.4 \pm 1.0$ \\
\hline LP-DOTAP/Sito & DOTAP:Sito = 1:1 & $127.6 \pm 0.9$ & $0.25 \pm 0.02$ & $44.3 \pm 0.2$ & $174.5 \pm 1.3$ & $0.21 \pm 0.01$ & $33.9 \pm 0.6$ \\
\hline
\end{tabular}

${ }^{a}$ In water; ${ }^{b}$ charge ratio (+:-) of cationic lipid to siRNA phosphate $=4: 1$. Each value represents the mean \pm standard deviation $(\mathrm{n}=3)$. LP, liposome; Chol, cholesterol; Stig, stigmasterol; Ergo, ergosterol; Sito, $\beta$-sitosterol; PDI, polydispersity index; N.D., not determined; DDAB, dimethyldioctadecylammonium bromide; DOTAP, 1,2-dioleoyl-3-trimethylammonium-propane methyl sulfate salt; si, short interfering.
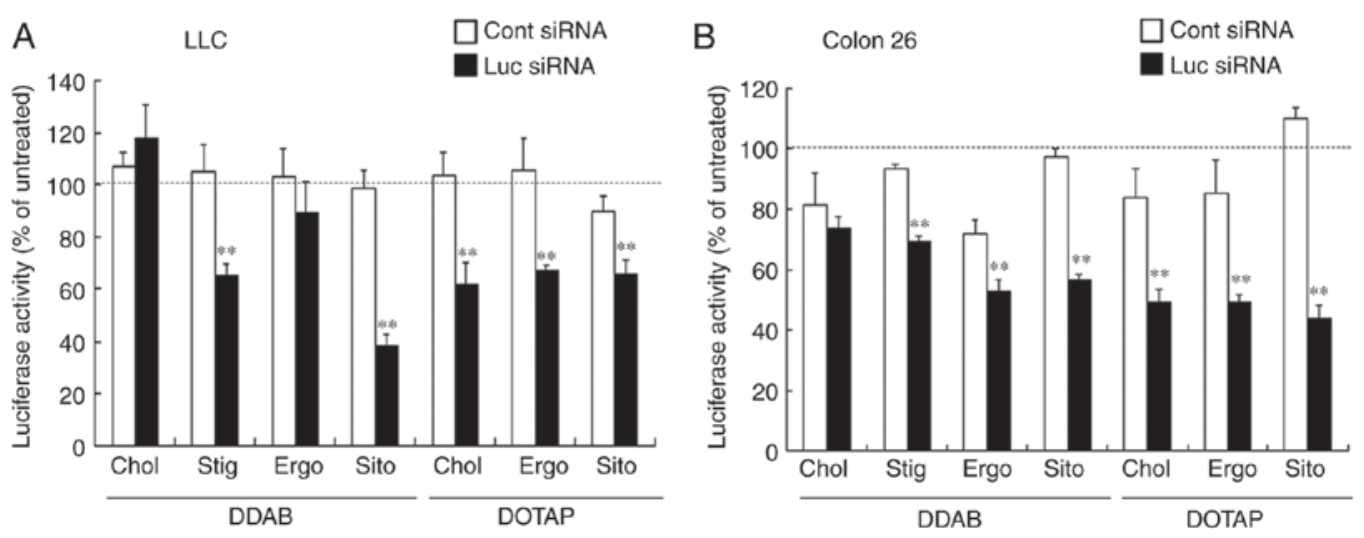

Figure 3. Effects of sterol derivatives in cationic liposomes on gene suppression in siRNA lipoplex transfected LLC-Luc and Colon 26-Luc cells. (A) LLC-Luc or (B) Colon 26-Luc cells were incubated for $48 \mathrm{~h}$ with $50 \mathrm{nM}$ Cont siRNA or Luc siRNA lipoplexes, then luciferase activity was assayed. Data are presented as the mean \pm standard deviation $(n=3) .{ }^{* *} \mathrm{P}<0.01$ vs. Cont siRNA. si, short interfering; lipoplex, cationic liposome complexes; DDAB, dimethyldioctadecylammonium bromide; DOTAP, 1,2-dioleoyl-3-trimethylammonium-propane methyl sulfate salt; Chol, cholesterol; Stig, stigmasterol; Ergo, ergosterol; Sito, $\beta$-sitosterol; Cont, control; Luc, luciferase.
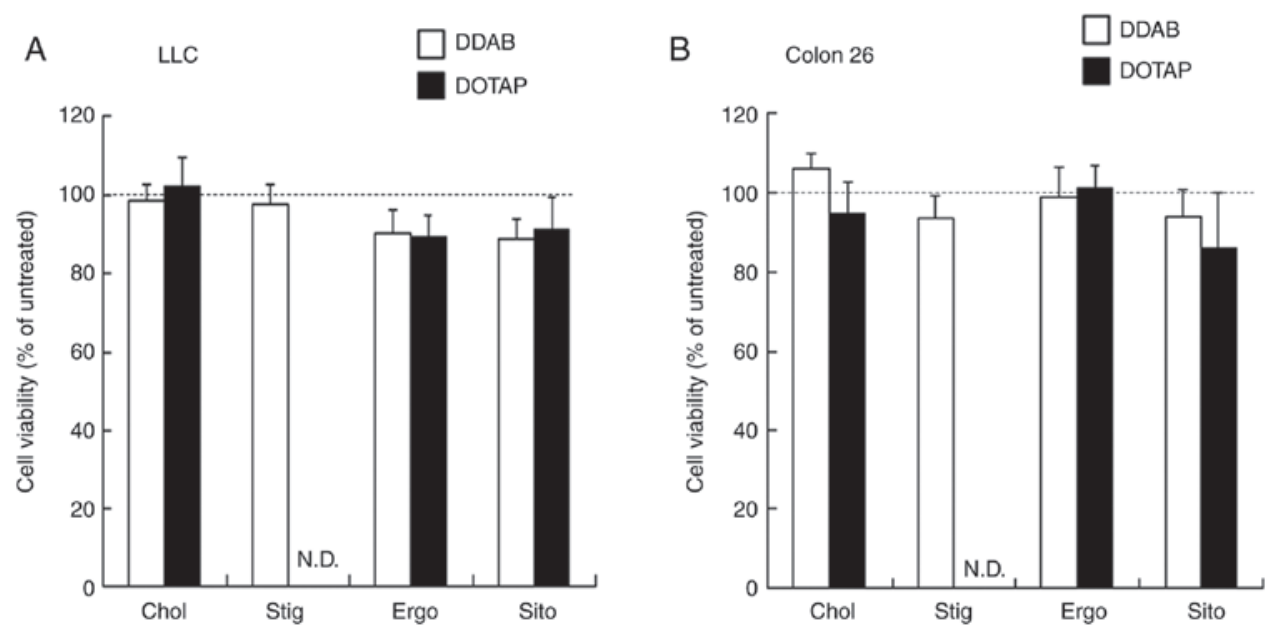

Figure 4. Effects of sterol derivatives in cationic liposomes on cell viability $24 \mathrm{~h}$ after transfection with siRNA lipoplexes into LLC-Luc or Colon 26-Luc cells. siRNA lipoplexes were added to (A) LLC-Luc or (B) Colon 26-Luc cells at $50 \mathrm{nM}$ siRNA. After a $24 \mathrm{~h}$ incubation period, cell viability (\%) was measured. Data are presented as the mean \pm standard deviation (A, n=7-8; B, n=6-7). N.D., not determined; DDAB, dimethyldioctadecylammonium bromide; DOTAP, 1,2-dioleoyl-3-trimethylammonium-propane methyl sulfate salt; Chol, cholesterol; Stig, stigmasterol; Ergo, ergosterol; Sito, $\beta$-sitosterol; lipoplex, cationic liposome complexes. 


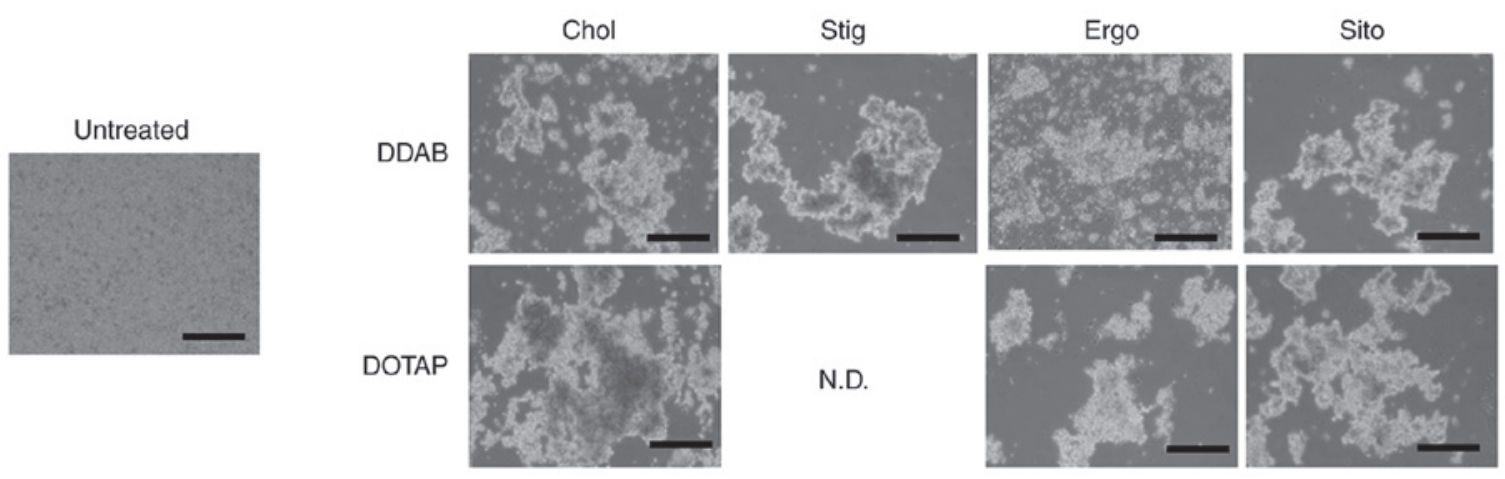

Figure 5. Effect of sterol derivatives in cationic liposomes on agglutination of siRNA lipoplexes with erythrocytes. Lipoplexes carrying $2 \mu \mathrm{g}$ siRNA were incubated with erythrocyte suspensions for $15 \mathrm{~min}$ at $37^{\circ} \mathrm{C}$, then assessed by phase contrast microscopy. DOTAP/Stig at a molar ratio of $1: 1$ aggregated during the preparation of cationic liposomes, so this formulation was excluded from further study. Scale bar, $200 \mu \mathrm{m}$. N.D., not determined; DDAB, dimethyldioctadecylammonium bromide; DOTAP, 1,2-dioleoyl-3-trimethylammonium-propane methyl sulfate salt; Chol, cholesterol; Stig, stigmasterol; Ergo, ergosterol; Sito, $\beta$-sitosterol; lipoplex, cationic liposome complexes.

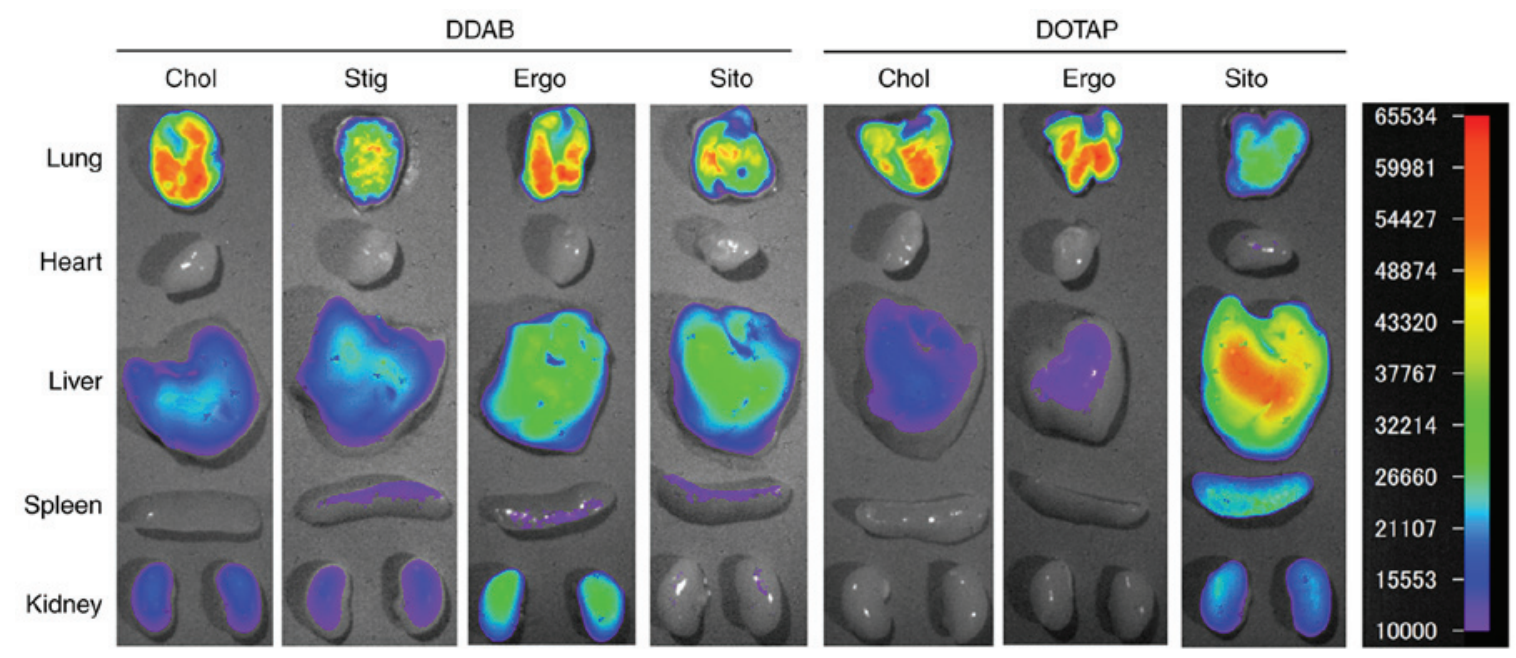

Figure 6. Ex vivo images of dissected tissues at $1 \mathrm{~h}$ after injections of siRNA lipoplexes into mice. siRNA lipoplexes with $20 \mu \mathrm{g}$ of Cy5.5-siRNA were administered intravenously into mice. The exposure time for the detection of Cy5.5 fluorescence was 5 sec. Red fluorescence indicates maximal intensity and purple fluorescence indicates minimal intensity. DDAB, dimethyldioctadecylammonium bromide; DOTAP, 1,2-dioleoyl-3-trimethylammonium-propane methyl sulfate salt; Chol, cholesterol; Stig, stigmasterol; Ergo, ergosterol; Sito, $\beta$-sitosterol; lipoplex, cationic liposome complexes.
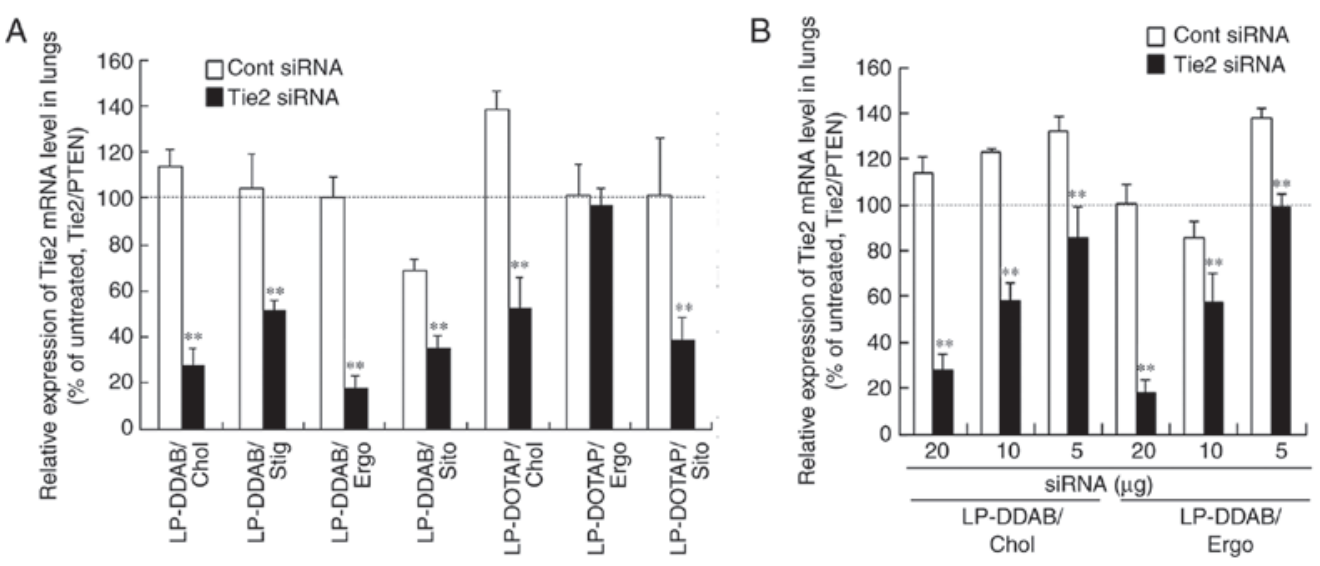

Figure 7. Effects of sterol derivatives in cationic liposomes on knockdown of Tie 2 mRNA expression in the lungs of mice systemically injected with siRNA lipoplexes carrying Tie2 siRNA. (A) Expression of Tie 2 mRNA in lungs was quantified relative to that of PTEN mRNA at $48 \mathrm{~h}$ after systemic administration of LP-DDAB/Chol, LP-DDAB/Stig, LP-DDAB/Ergo, LP-DDAB/Sito, LP-DOTAP/Chol, LP-DOTAP/Ergo and LP-DOTAP/Sito lipoplexes along with $20 \mu \mathrm{g}$ of Cont siRNA or Tie2 siRNA. (B) Tie2 mRNA expression in the lungs was quantified relative to that of PTEN mRNA at $48 \mathrm{~h}$ after systemic administration of LP-DDAB/Chol and LP-DDAB/Ergo lipoplexes with 5, 10 or $20 \mu \mathrm{g}$ of Cont siRNA or Tie2 siRNA. Expression (\%) of Tie2 mRNA was calculated relative to that of non-transfected lungs. Data are presented as the mean \pm standard deviation $(\mathrm{n}=3-4) .{ }^{* *} \mathrm{P}<0.01$ vs. Cont siRNA. LP; liposome, Chol; cholesterol, Stig; stigmasterol, Ergo; ergosterol, Sito; $\beta$-sitosterol; DDAB, dimethyldioctadecylammonium bromide; DOTAP, 1,2-dioleoyl-3-trimethylammonium-propane methyl sulfate salt; si, short interfering; Tie2, angiopoietin-1 receptor; Cont, control; lipoplex, cationic liposome complexes. 
Table II. Summary of gene-silencing effect by siRNA lipoplexes.

\begin{tabular}{lccc}
\hline Liposome & $\begin{array}{c}\text { Gene-silencing efficacy } \\
\text { in LLC cells }\end{array}$ & $\begin{array}{c}\text { Gene-silencing } \\
\text { efficacy in Colon26 cells }\end{array}$ & $\begin{array}{c}\text { Gene-silencing } \\
\text { efficacy in lungs }\end{array}$ \\
\hline LP-DDAB/Chol & - & - & +++ \\
LP-DDAB/Stig & + & - & ++ \\
LP-DDAB/Ergo & - & + & ++ \\
LP-DDAB/Sito & ++ & + & + \\
LP-DOTAP/Chol & + & + & + \\
LP-DOTAP/Ergo & + & ++ & ++ \\
LP-DOTAP/Sito & - & +
\end{tabular}

Gene-silencing efficacy: +++, >70\% knockdown; ++, 50-70\% knockdown; +: 30-50\% knockdown; -, <30\% knockdown, compared with control siRNA. LP, liposome; Chol, cholesterol; Stig, stigmasterol; Ergo, ergosterol; Sito, $\beta$-sitosterol; DDAB, dimethyldioctadecylammonium bromide; DOTAP, 1,2-dioleoyl-3-trimethylammonium-propane methyl sulfate salt; si, short interfering.

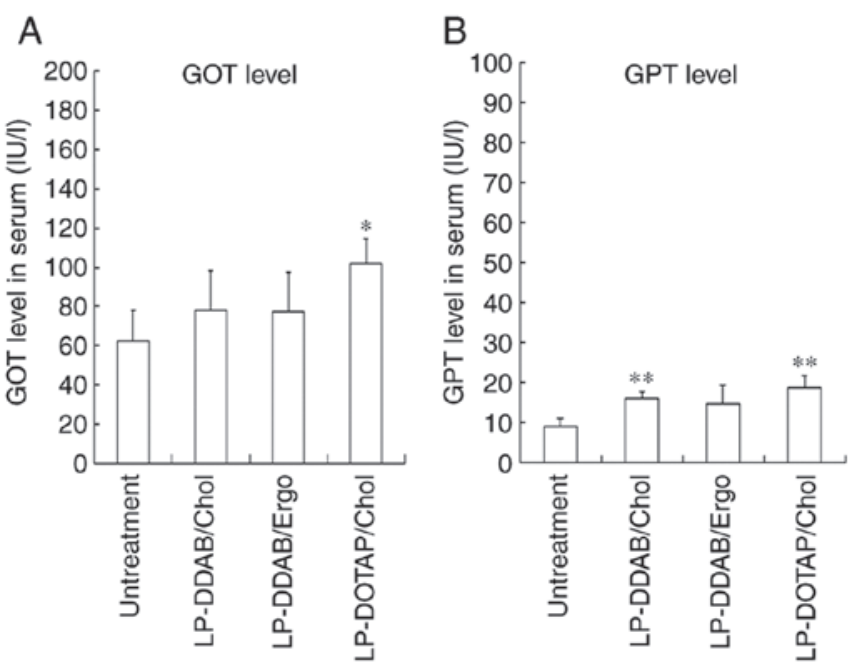

Figure 8. Liver toxicity after systemic injection of siRNA lipoplexes into mice. LP-DDAB/Chol, LP-DDAB/Ergo and LP-DOTAP/Chol lipoplexes with $20 \mu \mathrm{g}$ Cont siRNA were injected into the lateral tail veins of mice, after which concentrations of (A) GOT and (B) GPT in blood were measured $24 \mathrm{~h}$ later. Values are shown as means \pm standard deviation $(n=4)$. ${ }^{*} \mathrm{P}<0.05$ and ${ }^{* *} \mathrm{P}<0.01$ vs. untreated control. LP, liposome; Chol, cholesterol; Ergo, ergosterol; DDAB, dimethyldioctadecylammonium bromide; DOTAP, 1,2-dioleoyl-3-trimethylammonium-propane methyl sulfate salt; si, shor interfering; GOT, aspartate aminotransferase; GPT, alanine aminotransferase; lipoplex, cationic liposome complexes.

measured the gene-silencing effect of Tie 2 mRNA in the lungs of mice $48 \mathrm{~h}$ given a single systemic injection of lipoplexes carrying $20 \mu \mathrm{g}$ Tie 2 siRNA (Fig. 7A). Injected LP-DDAB/Chol, LP-DDAB/Stig, LP-DDAB/Ergo, and LP-DDAB/Sito lipoplexes with Tie2 siRNA significantly suppressed Tie2 mRNA levels $(\sim 76,51,82$ and 49\%, respectively, vs. Cont siRNA; Figs. 7A and S1).Injected LP-DOTAP/Chol and LP-DOTAP/Sito lipoplexes with Tie2 siRNA significantly knocked down Tie2 mRNA in the lungs by $\sim 62$ and $62 \%$, respectively, compared with Cont siRNA, whereas LP-DOTAP/Ergo lipoplexes with Tie2 siRNA did not (Figs. 7A and S1). Among the cationic liposomes, LP-DDAB/Chol and LP-DDAB/Ergo exhibited the highest gene silencing effect in the lungs. Injected LP-DDAB/Chol or LP-DDAB/Ergo lipoplexes carrying $10 \mu \mathrm{g}$ of Tie 2 siRNA significantly knocked down Tie 2 mRNA expression in mouse lungs by $\sim 40 \%$ vs. compared with untreated lungs; Fig. 7B).

Neutral helper lipids with membrane fluidity and rigidity in cationic liposomes affect gene silencing. The silencing efficiency of siRNA lipoplexes in vivo (Fig. 7A) and in vitro (Fig. 3) did not correlate. Including Chol in DDAB-based cationic liposomes decreased gene knockdown efficacy in vitro, but increased accumulation and gene knockdown efficacy in the lungs. siRNA lipoplexes stabilized with Chol might form stable siRNA lipoplex-erythrocyte aggregates, that are efficiently captured by lung capillaries, resulting in abundant siRNA transfection into the pulmonary endothelium. Regardless of the sterol derivative, DDAB-based cationic liposomes transfected in vivo induced significant gene silencing in the lungs. In contrast, DOTAP-based cationic liposomes elicited significant gene-silencing effects in the lungs only when Chol or Sito were included. These findings indicated that the degree of saturation of alkyl chains and/or linkers between the head group and alkyl chains in cationic lipids affects the optimal combination of sterol derivatives and thus the ability of systemically injected siRNA lipoplexes to knock down genes in the lungs. Among the cationic liposomes examined herein, LP-DDAB/Chol and LP-DDAB/Ergo knocked down genes in the lungs with $70-80 \%$ efficiency (Figs. 7 and S1, and Table II).

Side effects in the liver. Injected plasmid DNA (pDNA) lipoplexes containing the cationic lipid DOTAP induces toxic effects in the liver, but not in the lungs (27). Therefore, we assessed GOT and GPT levels in serum after injecting mice with LP-DDAB/Chol, LP-DDAB/Ergo, and LP-DOTAP/Chol lipoplexes to evaluate hepatic toxicity (Fig. 8). None of these siRNA lipoplexes largely increased serum GOT and GPT levels at $24 \mathrm{~h}$. These results suggested that the systemic administration of LP-DDAB/Chol, LP-DDAB/Ergo, and LP-DOTAP/Chol lipoplexes did not induce high side effects in terms of hepatotoxicity.

A single injection of DACC lipoplexes $(2.8 \mathrm{mg}$ Tie2 siRNA/kg) comprising the cationic lipid $\beta$-L-arginyl-2,3L-diaminopropionic acid- $N$-palmityl- $N$-oleyl-amide trihydrochloride (AtuFECT01), decreases Tie2 mRNA levels by 
$>80 \%$ after 3 days (3). We showed that a single injection of LP-DDAB/Chol and LP-DDAB/Ergo lipoplexes carrying Tie2 siRNA $(1 \mathrm{mg} / \mathrm{kg})$ reduced the expression of Tie 2 mRNA in the lungs by $70-80 \%$ within 2 days (Fig. 7A), indicating that LP-DDAB/Chol and LP-DDAB/Ergo lipoplexes carrying lower doses of siRNA can efficiently knock down the expression of a targeted gene in the lung endothelium. Among the sterol derivatives, ergosterol has anti-tumor and anti-angiogenic activities $(28,29)$. Therefore, LP-DDAB/Ergo might be an effective siRNA vector for treating lung tumors. Our findings showed that cationic DDAB-based liposomes combined with sterol derivatives have potential as vectors for delivering siRNA therapeutics to the lungs.

We examined the effects of sterol derivatives in cationic liposomes on siRNA biodistribution and gene knockdown in the lungs of mice systemically injected with siRNA lipoplexes. DDAB-based liposomal formulations including Chol or Ergo effectively silenced genes in the lungs of mice. This study provides valuable information about the optimal combination of cationic lipids and sterol derivatives in liposomal formulations for optimal siRNA delivery to the lungs.

\section{Acknowledgements}

Not applicable.

\section{Funding}

No funding was received.

\section{Availability of data and materials}

The datasets used and/or analyzed during the current study are available from the corresponding author on reasonable request.

\section{Authors' contributions}

YH conceived and designed the study. HS, TO and KIO conducted experiments. YH and HS confirm the authenticity of all the raw data. YH wrote the manuscript. All authors have read and approved the final manuscript.

\section{Ethics approval and consent to participate}

The Institutional Animal Care and Use Committee of Hoshi University approved the animal experiments of the present study (approval no. 20-018).

\section{Patient consent for publication}

Not applicable.

\section{Competing interests}

The authors declare that they have no competing interests.

\section{References}

1. Merkel OM, Rubinstein I and Kissel T: siRNA delivery to the lung: What's new? Adv Drug Deliv Rev 75: 112-128, 2014.
2. Fujita Y, Takeshita F, Kuwano K and Ochiya T: RNAi Therapeutic platforms for lung diseases. Pharmaceuticals (Basel) 6: 223-250, 2013.

3. Fehring V, Schaeper U, Ahrens K, Santel A, Keil O, Eisermann M, Giese K and Kaufmann J: Delivery of therapeutic siRNA to the lung endothelium via novel Lipoplex formulation DACC. Mol Ther 22: 811-820, 2014.

4. Garbuzenko OB, Saad M, Betigeri S, Zhang M, Vetcher AA Soldatenkov VA, Reimer DC, Pozharov VP and Minko T: Intratracheal versus intravenous liposomal delivery of siRNA, antisense oligonucleotides and anticancer drug. Pharm Res 26: 382-394, 2009

5. Merkel OM and Kissel T: Nonviral pulmonary delivery of siRNA. Acc Chem Res 45: 961-970, 2012.

6. McCaskill J, Singhania R, Burgess M, Allavena R, Wu S, Blumenthal A and McMillan NA: Efficient biodistribution and gene silencing in the lung epithelium via intravenous liposomal delivery of siRNA. Mol Ther Nucleic Acids 2: e96, 2013.

7. Kuruba R, Wilson A, Gao X and Li S: Targeted delivery of nucleic-acid-based therapeutics to the pulmonary circulation. AAPS J 11: 23-30, 2009.

8. Hattori Y, Nakamura M, Takeuchi N, Tamaki K, Shimizu S, Yoshiike Y, Taguchi M, Ohno H, Ozaki KI and Onishi H: Effect of cationic lipid in cationic liposomes on siRNA delivery into the lung by intravenous injection of cationic lipoplex. J Drug Target 27: 217-227, 2019.

9. Hattori Y, Tamaki K, Ozaki K, Kawano K and Onishi H: Optimized combination of cationic lipids and neutral helper lipids in cationic liposomes for siRNA delivery into the lung by intravenous injection of siRNA lipoplexes. J Drug Deliv Sci Technol 52: 1042-1050, 2019.

10. Hattori Y, Shimizu S, Ozaki KI and Onishi H: Effect of cationic lipid type in folate-PEG-modified cationic liposomes on folate receptor-mediated siRNA transfection in tumor cells. Pharmaceutics 11: 11, 2019.

11. Hattori Y, Nakamura A, Arai S, Kawano K, Maitani Y and Yonemochi E: siRNA delivery to lung-metastasized tumor by systemic injection with cationic liposomes. J Liposome Res 25: 279-286, 2015.

12. Hattori Y, Arai S, Kikuchi T, Ozaki KI, Kawano K and Yonemochi E: Therapeutic effect for liver-metastasized tumor by sequential intravenous injection of anionic polymer and cationic lipoplex of siRNA. J Drug Target 24: 309-317, 2016.

13. Aleku M, Schulz P, Keil O, Santel A, Schaeper U, Dieckhoff B, Janke O, Endruschat J, Durieux B, Röder N, et al: Atu027, a liposomal small interfering RNA formulation targeting protein kinase N3, inhibits cancer progression. Cancer Res 68: 9788-9798, 2008.

14. Hattori Y, Tamaki K, Sakasai S, Ozaki KI and Onishi H: Effects of PEG anchors in PEGylated siRNA lipoplexes on in vitro gene silencing effects and siRNA biodistribution in mice. Mol Med Rep 22: 4183-4196, 2020.

15. Hattori Y, Nakamura T, Ohno H, Fujii N and Maitani Y: siRNA delivery into tumor cells by lipid-based nanoparticles composed of hydroxyethylated cholesteryl triamine. Int J Pharm 443: 221-229, 2013.

16. Sato A, Ohtsuki M, Hata M, Kobayashi E and Murakami T: Antitumor activity of IFN-lambda in murine tumor models. J Immunol 176: 7686-7694, 2006.

17. Oku T, Ando Y, Ogura M and Tsuji T: Development of splice variant-specific monoclonal antibodies against human alpha3 integrin. Monoclon Antib Immunodiagn Immunother 35: 12-17, 2016.

18. Oku T, Shimada K, Kenmotsu H, Ando Y, Kurisaka C, Sano R, Tsuiji M, Hasegawa S, Fukui T and Tsuji T: Stimulation of peritoneal mesothelial cells to secrete matrix metalloproteinase-9 (MMP-9) by TNF-alpha: A role in the invasion of gastric carcinoma cells. Int J Mol Sci 19: 19, 2018.

19. Hattori Y, Nakamura A, Hanaya S, Miyanabe Y, Yoshiike Y, Kikuchi T, Ozaki K and Onishi H: Effect of chondroitin sulfate on siRNA biodistribution and gene silencing effect in mice after injection of siRNA lipoplexes. J Drug Deliv Sci Technol 41: 401-409, 2017.

20. Livak KJ and Schmittgen TD: Analysis of relative gene expression data using real-time quantitative PCR and the 2(-Delta Delta C(T)) method. Methods 25: 402-408, 2001.

21. Hu Z, He B, Ma L, Sun Y, Niu Y and Zeng B: Recent advances in ergosterol biosynthesis and regulation mechanisms in Saccharomyces cerevisiae. Indian J Microbio 57: 270-277, 2017.

22. Benveniste P: Biosynthesis and accumulation of sterols. Annu Rev Plant Biol 55: 429-457, 2004. 
23. Eliyahu H, Servel N, Domb AJ and Barenholz Y:Lipoplex-induced hemagglutination: Potential involvement in intravenous gene delivery. Gene Ther 9: 850-858, 2002.

24. Simberg D, Weisman S, Talmon Y, Faerman A, Shoshani T and Barenholz Y: The role of organ vascularization and lipoplex-serum initial contact in intravenous murine lipofection. J Biol Chem 278: 39858-39865, 2003.

25. Loughna S and Sato TN: Angiopoietin and Tie signaling pathways in vascular development. Matrix Biol 20: 319-325, 2001.

26. van der Heijden M, van Nieuw Amerongen GP, Chedamni S, van Hinsbergh VW and Johan Groeneveld AB: The angiopoietin-Tie2 system as a therapeutic target in sepsis and acute lung injury. Expert Opin Ther Targets 13: 39-53, 2009.

27. Loisel S, Le Gall C, Doucet L, Ferec C and Floch V: Contribution of plasmid DNA to hepatotoxicity after systemic administration of lipoplexes. Hum Gene Ther 12: 685-696, 2001.
28. Chen S, Yong T, Zhang Y, Su J, Jiao C and Xie Y: Anti-tumor and Anti-angiogenic Ergosterols from Ganoderma lucidum. Front Chem 5: 85, 2017.

29. Takaku T, Kimura Y and Okuda H: Isolation of an antitumor compound from Agaricus blazei Murill and its mechanism of action. J Nutr 131: 1409-1413, 2001

c) (i) () $९$ This work is licensed under a Creative Commons Attribution-NonCommercial-NoDerivatives 4.0 International (CC BY-NC-ND 4.0) License. 